\title{
HOGARES DE LA FRONTERA NORTE DE MÉXICO. CAMBIOS SOCIO- DEMOGRÁFICOS EN EL MARCO FAMILIAR TRADICIONAL
}

\section{HOMES OF THE NORTHERN BORDER OF MEXICO. SOCIODEMOGRAPHIC CHANGES IN THE TRADITIONAL FAMILY SETTING}

\author{
Humberto González Galbán \\ Adriana Ortiz Peredo \\ Colegio de la Frontera Norte, Tijuana, Baja California. México/Mexico \\ hggalban@colef.mx \\ aortiz@ colef.mx
}

Recibido/Received: 18/08/2017

Modificado/Modified: 25/09/2017

Aceptado/Accepted: 05/10/2017

\section{RESUMEN}

Comúnmente se plantea en la literatura especializada que en el marco de la institución familiar se opera la reproducción cotidiana, generacional y social de la población, lo cual puede ser motivo de controversia tanto por la heterogeneidad de tipos de familias existentes como por las transformaciones que se están experimentando en las mismas, lo que amerita ser oportunamente evidenciado en interrelación con el entorno existente y dimensionado acorde la trascendencia atribuida a esta organización social. Para la investigación de los aspectos señalados en el actual artículo se analizan indicadores que hacen hincapié básicamente en los jóvenes de la frontera norte de México y las problemáticas familiares en torno a los mismos. Como resultados se destaca una mayor proporción de hogares no nucleares donde residen jóvenes que en el resto del país. También es destacable una mayor actividad sexual fuera de uniones estables sin contar estos con preparación previa para ello.

PALABRAS CLAVE

Familias; jóvenes; frontera norte de México.

\section{SUMARIO}

1. Introducción. 2. Características estructurales y funcionales de la familia en el contexto sociodemográfico de la frontera norte de México. 3. Tamaño y estructura de los hogares con jóvenes en la frontera norte de México. 4. Formación y desarrollo de los arreglos familiares. 5. Arreglos conyugales en el marco de los que se lleva a cabo la sexualidad y la reproducción. 6. Indicadores de cambios en la importancia de la familia. 7. Comportamiento sexual y reproducción. 8. Conclusiones. Bibliografía.

\footnotetext{
ABSTRACT

Commonly arises in the literature that in the context of the family institution operates daily, generational and social reproduction of the population, which however is controversial at present both the diversity of existing types of families as the transformations that they are experiencing. It deserves to be duly evidenced in interaction with the existing environment and sized according to the importance attributed
} 
to this social organization. For the investigation of the aspects indicated in the present article, we analyze indicators that emphasize basically the young people of the northern border of Mexico and the familiar problems around them. The results show a higher proportion of non-nuclear households where young people live than in the rest of the country. It is also remarkable a greater sexual activity outside of stable unions without counting these with previous preparation for it.

\section{KEYWORDS}

Families; young, northern border of Mexico.

\section{CONTENTS}

1. Introduction. 2. Structural and functional characteristics of the family in the sociodemographic context of the northern border of Mexico. 3. Size and structure of homes with young people on the northern border of Mexico. 4. Training and development of family arrangements. 5. Conjugal arrangements within the framework of sexuality and reproduction. 6. Signs of changes in the meaning of family. 7. Sexual Behavior and reproduction. 8. Conclusions. References.

"El lugar donde nacen los niños y mueren los hombres, donde la libertad y el amor florecen, no es una oficina ni un comercio ni una fábrica. Ahí veo yo la importancia de la familia”. Gilbert Keith Chesterton

\section{INTRODUCCIÓN}

En la frontera norte de México se presentan peculiaridades de interés generadas tanto por su cercanía e importante interrelación con los Estados Unidos de América, como por el intenso proceso migratorio al que está vinculada básicamente con la población procedente del resto del país, o sea, ambos procesos tan diversos ejercen su influencia al unísono en esta región, de donde puede surgir una organización poblacional con características sociales y demográficas diferentes.

Relacionado a ello se debe destacar los aspectos demográficos o sociales que pueden haber tenido un peso importante en la explicación de los cambios poblacionales de los países socioeconómicamente avanzados, como las características estructurales y funcionales de los hogares, la nupcialidad o arreglos conyugales en el marco de los que se lleva a cabo la sexualidad y/o la reproducción, la participación en el mercado laboral y la incidencia de todo ello en los cambios familiares, aspectos que son insuficientemente considerados en los análisis realizados en países en vías de desarrollo. Por lo tanto, el objeto de estudio de esta investigación, sugiere la necesidad de llevar a cabo estudios de dichos cambios familiares, particularmente en regiones caracterizadas al mismo tiempo por una importante afluencia de migrantes, como puede ser la frontera norte de México.

Acorde a ello usualmente se hace referencia a los hogares como el lugar donde una persona o un grupo de personas, emparentadas o no, habitan (Ver Figura 1). En base a la estructura o relación de los miembros que integran dichos hogares, así como a apreciaciones legales o de otro tipo cada país clasifica los hogares existentes en los mismos. En México éstos serían como se indica en la figura 1.

$\mathrm{Al}$ interior de los hogares aparece la familia como la institución en el marco de la que se produce la reproducción biológica y social de la población, donde dominan las relaciones de solidaridad, no exentas de conflictos entre sus miembros, lo que repercute en nuevas formas de organización de las personas y en la transformación de características de la misma. No 
obstante que, la familia puede continuar representando en general el entorno mediato en el que se desarrollan los individuos a lo largo de sus ciclos de vida, donde además de reproducirse las relaciones sociales, se gestan los cambios demográficos, sus características pueden estar variando en sentido y magnitud.

Figura 1. Clasificación de tipos de hogares en México
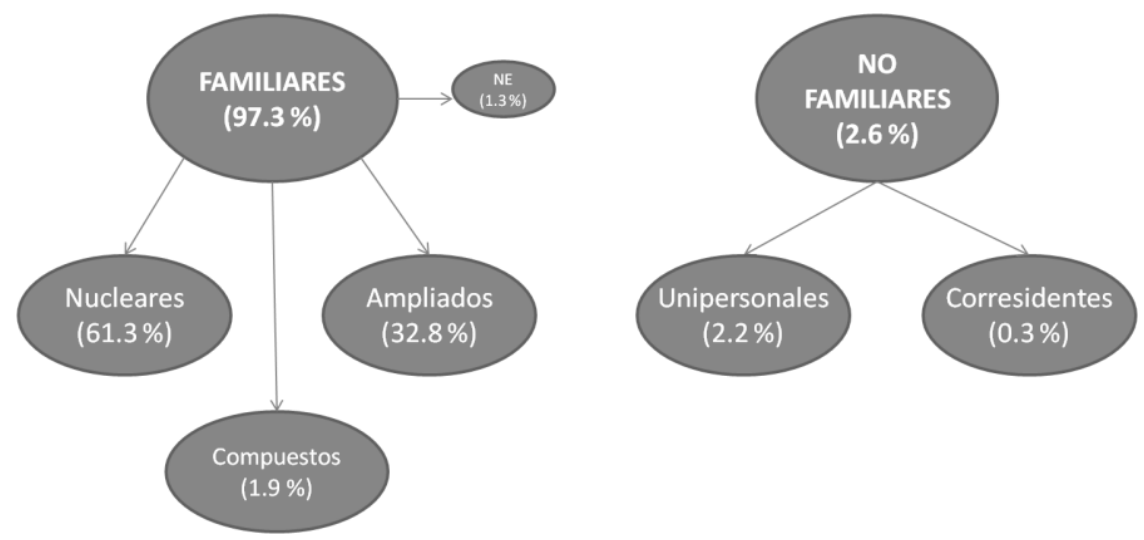

Fuente: Elaboración propia con base en la clasificación dada por el INEGI

En los hogares se desarrollan lazos de pareja, ya sea a través del mantenimiento de relaciones sexuales y/o afectivas que dan origen a la familia. En la región de la frontera norte de México, se manifiesta una transición demográfica relativamente avanzada, lo que se evidencia en la forma en que se construye y desarrolla la familia, con una nupcialidad más marcada por la convivencia e inestabilidad y precedida por un mayor período de relaciones sexuales de las jóvenes -sin una preparación adecuada para ello- antes de la unión conyugal, así como una descendencia, que aunque se ha hecho significativamente menor, aparece desplazada a etapas más tempranas en el ciclo de vida de la mujer. Estos y otros aspectos serán analizados en el actual artículo, que tiene como objetivo central aportar elementos que permitan responder a la pregunta acerca de la transformación de la familia en la frontera norte de México, lo que se sustenta en diferentes fuentes de información estadística generadas por el Instituto Nacional de Geografía e Informática (INEGI) en años recientes, así como en diversos trabajos documentales desarrollados por investigadores.

Entre la información estadística generada por el INEGI aquí utilizada se destaca la Encuesta de la Dinámica Demográfica (ENADID), 2014, la cual está estructurada en cuestionarios sobre tópicos sobre hogares y otro con módulos que contienen preguntas sobre características socioeconómicas y demográficas de los residentes tales como la fecundidad, la nupcialidad y otras temáticas afines a lo aquí analizado. Otra fuente de información puesta a disposición por el INEGI fue la ENADID, 2009 y la Encuesta Intercensal 2015 y por otras instituciones de reconocida confiabilidad como El Colegio de La Frontera Norte que elaboró la Encuesta de Salud Reproductiva en los Adolescentes de Baja California del año 2006 que contiene gran riqueza sobre novedosos datos, sobre los jóvenes y sus familias en el norte del país. 


\section{CARACTERÍSTICAS ESTRUCTURALES Y FUNCIONALES DE LA FAMILIA EN EL CONTEXTO SOCIODEMOGRÁFICO DE LA FRONTERA NORTE DE MÉXICO}

Para la comprensión de los cambios en la estructura, desarrollo y funciones de la familia, es necesario tomar en cuenta el proceso de la transición demográfica en el contexto regional considerado, que en este caso está limitado a la frontera norte de México, el que presenta peculiaridades vinculadas a su ubicación geográfica y a un desarrollo socioeconómico relacionado en buena medida a los Estados Unidos, como fue antes referido.

A tal fin se puede comenzar destacando el elevado crecimiento poblacional experimentado durante el pasado siglo XX en esta región. El referido crecimiento poblacional respondió a la fuerte inmigración que experimentó esta zona del país condicionada por la demanda de mano de obra, básicamente en las industrias maquiladoras de exportación, lo que generó importantes saldos migratorios positivos.

También como efectos de la acentuada inmigración se encuentra la composición por edad y sexo de los pobladores de la frontera, lo que mantuvo la estructura más joven a la esperada, -con un retraso del proceso de envejecimiento poblacional- en correspondencia con los relativamente bajos niveles de fecundidad registrados.

Gráfico 1. Pirámides poblacionales, Zona fronteriza y Nacional

\begin{tabular}{|c|c|}
\hline $\begin{array}{l}\text { Zona fronteriza, pirámide de población } \\
2015\end{array}$ & $\begin{array}{l}\text { México, pirámide de población } \\
2015\end{array}$ \\
\hline 100y más & 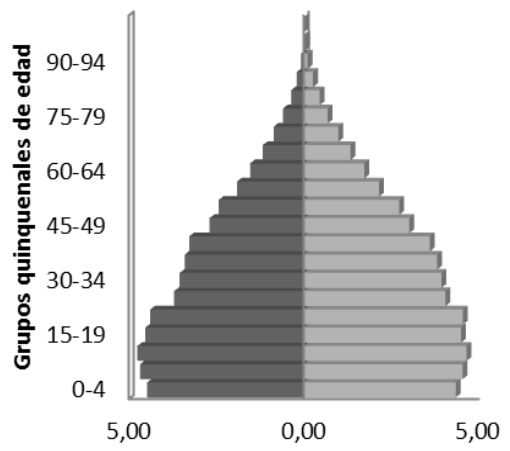 \\
\hline
\end{tabular}

Fuente: Elaboración propia con datos de INEGI, Encuesta Intercensal 2015

No obstante, si analizamos detenidamente el Gráfico 1, podemos observar que la distribución poblacional presenta importantes variaciones. En la zona fronteriza, la población menor de 20 años tiene una menor representación que a nivel nacional, mientras que la población mayor de 60 años tiene una mayor presencia. Uno de los cambios esenciales tiene que ver con la disminución en las tasas de fecundidad, por ejemplo, en 2009 Baja California presentaba una de las más altas tasas de fecundidad adolescente, sin embargo, los resultados de la ENADID 2014 (54.8 \%) nos señalan que la situación al respecto ha cambiado, al ocupar los últimos lugares a nivel nacional, por lo tanto, resulta interesante observar la actual estructura poblacional de la zona fronteriza, ya que dista del comportamiento presentado en 
años anteriores, caracterizado por tener un alto porcentaje de población joven y en edad de trabajar.

Por otra parte, las tasas de migración en los estados fronterizos también revelan una disminución considerable, la entidad fronteriza que mayor variación presentó de 2010 a 2015 fue Tamaulipas, con un saldo neto migratorio de (-2.44), seguida de Chihuahua (-0.86).

\section{TAMAÑO Y ESTRUCTURA DE LOS HOGARES CON JÓVENES EN LA FRONTERA NORTE DE MÉXICO}

Como preámbulo de la temática referida se afirma que una forma de analizar los arreglos familiares que se pueden estar presentando en la zona norte son a través de las características de los hogares de los jóvenes en esta frontera del país. Al respecto se puede comenzar planteando que en México existen 31.9 millones de hogares y en 14.9 de ellos reside algún joven, lo que representa 66.0 por ciento del total de unidades domésticas. Esta proporción en la frontera norte es ligeramente superior $(66.8 \%)$.

El tamaño promedio de los hogares con jefes jóvenes del país es de 3.4 personas, cifra que se reduce ligeramente a 3.3 en la frontera norte (Gráfico 2). La composición de los hogares en la zona norte se encuentra más diferenciada, debido a que la proporción de hogares nucleares se presenta en mayor grado que a nivel nacional. Situación similar muestran los hogares compuestos y unipersonales.

En relación a la edad y sexo de los jefes de hogar, llama la atención el hecho de la elevada proporción de hogares con jefes jóvenes en la frontera, donde la proporción de los mismos alcanza 12.6 por ciento, contra 11.7 por ciento a nivel nacional.

Gráfico 2. Distribución de los hogares con jefes jóvenes, según número de integrantes de los mismos. Nacional, Frontera Norte y Resto de México

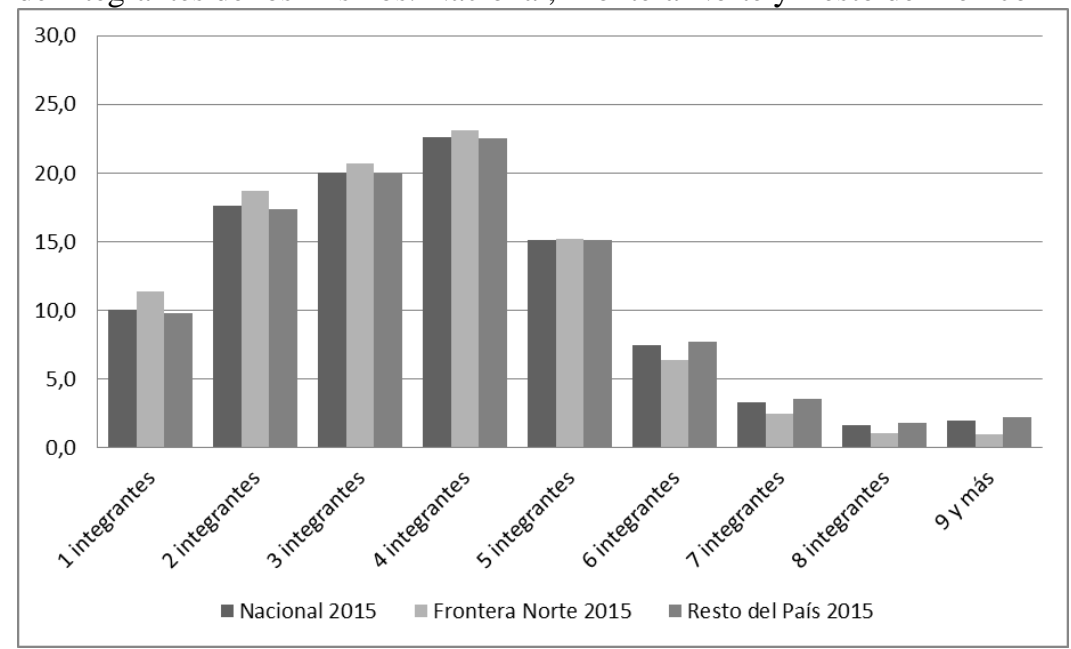

Fuente: INEGI, 2015, Encuesta Intercensal

También resulta interesante el que a nivel de país, del total de hogares con jóvenes, 9.0 por ciento están encabezados por hombres, mientras que 2.7 por ciento tienen al frente a una mujer. Esta inequidad de género se presenta un poco más atenuada en la frontera norte, 
donde se observa un ligero aumento en la presencia de jefatura femenina juvenil que a nivel nacional - $2.8 \%$ Vs. $2.7 \%$ - respectivamente (Cuadro 1).

Cuadro 1. Porcentaje de hogares donde residen jefes de vivienda jóvenes (12-29), según entidad fronteriza y el resto del país.

\begin{tabular}{|l|c|c|c|c|c|c|c|c|c|}
\hline $\begin{array}{l}\text { Tipo de } \\
\text { hogar }\end{array}$ & Nacional & $\begin{array}{l}\text { Baja } \\
\text { California }\end{array}$ & Coahuila & Chihuahua & $\begin{array}{l}\text { Nuevo } \\
\text { León }\end{array}$ & Sonora & Tamaulipas & $\begin{array}{l}\text { Frontera } \\
\text { Norte }\end{array}$ & $\begin{array}{l}\text { Resto } \\
\text { del } \\
\text { país }\end{array}$ \\
\hline Nuclear & 69,2 & 63,8 & 74,4 & 68,7 & 64,2 & 63,6 & 67,3 & 66,7 & 69,9 \\
\hline Ampliado & 15,4 & 17,6 & 14,4 & 14,2 & 18,4 & 16,5 & 18,5 & 16,8 & 15,0 \\
\hline Compuesto & 1,1 & 1,6 & 0,8 & 1,2 & 2,0 & 1,3 & 0,9 & 1,4 & 1,0 \\
\hline $\begin{array}{l}\text { Unipersonal } \\
\text { Corresidente }\end{array}$ & 3,1 & 12,9 & 6,4 & 9,7 & 8,8 & 12,6 & 9,4 & 10,0 & 9,4 \\
\hline $\begin{array}{l}\text { No } \\
\text { especificado }\end{array}$ & 1,7 & 0,9 & 1,6 & 3,4 & 1,9 & 1,8 & 1,8 & 1,9 & 1,6 \\
\hline $\begin{array}{l}\text { Hogares con } \\
\text { jefes jóvenes }\end{array}$ & 11,6 & 14,1 & 12,1 & 13,2 & 11,8 & 12,2 & 12,2 & 12,5 & 11,4 \\
\hline $\begin{array}{l}\text { Hogares con } \\
\text { jefes jóvenes } \\
\text { hombres }\end{array}$ & 9,0 & 10,2 & 10,2 & 10,0 & 9,7 & 8,7 & 9,4 & 9,7 & 8,8 \\
\hline $\begin{array}{l}\text { Hogares con } \\
\text { jefes jóvenes } \\
\text { mujeres }\end{array}$ & 2,7 & 3,8 & 2,0 & 3,2 & 2,1 & 3,4 & 2,8 & 2,8 & 2,6 \\
\hline
\end{tabular}

Fuente: INEGI, 2015. Encuesta Intercensal

Una peculiaridad de esta frontera es que en los últimos años se está manifestando un incremento de los hogares dirigidos por mujeres con parejas. Ello puede obedecer, según algunos autores (Vargas Valle, y María Navarro, 2012), al desarrollo de procesos que condicionan la autonomía individual. En general, la jefatura femenina ha presentado un aumento de cuatro puntos porcentuales entre 2010 y 2015, así pasó de 24.6 por ciento a 29.0 por ciento (EIC; 2015).

\section{FORMACIÓN Y DESARROLLO DE LOS ARREGLOS FAMILIARES}

Entre los factores estructurales vinculados a los importantes cambios demográficos que se produjeron en los países más desarrollados, se señalan los relacionados al mejoramiento de la posición económica, política y social de la mujer, a la democratización de la vida familiar, y a la autonomía de las parejas. Ello se vincula al incremento en las posibilidades de empleo y del salario de las mujeres, equiparándose al de los hombres, ampliación de la educación para poder acceder en iguales condiciones que ellos al mercado de trabajo.

Todo lo referido incidió en la postergación del matrimonio. Estos cambios que propició la modernización de la sociedad, a su vez ejercieron su influencia en otros aspectos como son el incremento de los divorcios, la actividad sexual fuera del matrimonio con fines no reproductivos y el aumento de la importancia relativa de los nacimientos extramaritales.

La relación divorcios-matrimonios se ha incrementado de manera significativa en catorce años, dicha relación casi se ha triplicado pasando de 7.4 en 2000 a 19.6 en 2014, esto a nivel nacional, mientras que en la zona fronteriza dicha situación es aún más aguda, pasando de 
11.0 a 25.9, cabe destacar que entre los estados fronterizos, Coahuila registra la relación más alta (EIC, 2015).

Las condiciones en que se desarrolla la población parecen estar cambiando, lo que acerca a este país al escenario referido, emergiendo así otros modelos donde es necesario considerar que las relaciones sexuales preceden en buena medida a la primera unión conyugal, lo que hace más amplios los períodos de exposición al embarazo, condicionando de este modo el incremento de los nacimientos fuera de las uniones tradicionales de pareja (Quilodrán, 2000).

En este aspecto se destaca también que el incremento del tamaño del intervalo entre la primera relación sexual y la unión estable de pareja, si no se acompaña con un uso adecuado de anticonceptivos, puede dar pie a la elevación del número de nacimientos no planificados, así como de los abortos practicados en condiciones sanitarias inapropiadas, con implicaciones sociales y de salud negativas que pueden afectar básicamente a grupos más vulnerables, como pueden ser las jóvenes.

En cuanto al tipo de unión conyugal se aprecia que el matrimonio formal manifiesta el papel hegemónico en la conformación de las familias existentes tanto en la frontera norte como en el resto del país, sobrepasando con mucho a las uniones libres, sin embargo en el caso de una entidad del norte como es Baja California dicha supremacía se hace significativamente menor, ganando en importancia -con relación a las otras entidades fronterizas- las uniones libres (Ver Cuadro 2). De igual forma se hace evidente como predominan las uniones con relación a los matrimonios entre las mujeres más jóvenes, para las que los estados conyugales no solteros pueden originarse en períodos con cohabitaciones menos estable, formales, como forma de estabilización de dichas relaciones o de legitimación de embarazos no planificados.

Cuadro 2. Distribución de mujeres por tipo de relación de pareja, según entidad de residencia y edad

\begin{tabular}{|c|c|c|c|c|c|}
\hline \multirow{2}{*}{$\begin{array}{c}\text { Entidad } \\
\text { federativa }\end{array}$} & \multirow{2}{*}{$\begin{array}{c}\text { Edad agrupada } \\
\text { mujeres }\end{array}$} & $\begin{array}{c}\text { Unión } \\
\text { conyugal }\end{array}$ & $\begin{array}{c}\text { Alguna vez } \\
\text { unidas * }\end{array}$ & En matrimonio & \multirow{2}{*}{ Solteras } \\
\cline { 2 - 6 } Frontera Norte & $15-19$ & 10.8 & 1.1 & 3.7 & 84.3 \\
\cline { 2 - 6 } & $20-49$ & 20.4 & 10.7 & 47.4 & 21.5 \\
\hline \multirow{2}{*}{ Baja California } & $15-19$ & 10.4 & 1.3 & 2.1 & 86.1 \\
\cline { 2 - 6 } & $20-49$ & 25.7 & 12.2 & 39.8 & 22.3 \\
\hline \multirow{2}{*}{ Coahuila } & $15-19$ & 11.6 & 1.2 & 7.1 & 80.2 \\
\cline { 2 - 6 } & $20-49$ & 16.7 & 10.1 & 53.3 & 19.9 \\
\hline \multirow{2}{*}{ Chiuahua } & $15-19$ & 11.7 & 1.1 & 3.5 & 83.7 \\
\cline { 2 - 6 } & $20-49$ & 23.5 & 11.2 & 43.6 & 21.7 \\
\hline \multirow{2}{*}{ Nuevo León } & $15-19$ & 9.7 & 0.8 & 3.6 & 85.8 \\
\cline { 2 - 6 } & $20-49$ & 14.7 & 9.0 & 53.9 & 22.4 \\
\hline \multirow{2}{*}{ Sonora } & $15-19$ & 10.6 & 1.5 & 2.8 & 85.1 \\
\cline { 2 - 6 } & $20-49$ & 23.1 & 11.8 & 42.8 & 22.3 \\
\hline \multirow{2}{*}{ Tamaulipas } & $15-19$ & 11.4 & 1.1 & 3.6 & 83.8 \\
\cline { 2 - 6 } & $20-49$ & 21.4 & 11.0 & 47.5 & 20.0 \\
\hline \multirow{2}{*}{$\begin{array}{c}\text { Resto de } \\
\text { México }\end{array}$} & $15-19$ & 11.7 & 1.1 & 4.0 & 83.3 \\
\cline { 2 - 6 } & $20-49$ & 21.6 & 10.4 & 43.6 & 24.4 \\
\hline \multirow{2}{*}{ Nacional } & $15-19$ & 11.5 & 1.1 & 3.9 & 83.4 \\
\cline { 2 - 6 } & $20-49$ & 21.4 & 10.4 & 44.3 & 23.9 \\
\hline & $*$ Incluye divorciadas, separadas , viudas & & \\
\hline
\end{tabular}

Fuente: INEGI, 2015, Encuesta Intercensal 
La edad al comienzo de las uniones en el norte del país es relativamente elevada, con un valor que supera los 22 años, lo que no implica sin embargo una menor exposición al riesgo de un embarazo generalmente no deseado, ya que es en las edades más jóvenes cuando las relaciones sexuales comienzan -cada vez de forma más temprana- (Ver Cuadro 3) y donde no suelen planificarse adecuadamente los encuentros "amorosos", lo que se asocia generalmente al no uso de anticonceptivos efectivos, particularmente en las primeras relaciones, ya sea por problemas de acceso a dichos métodos o apreciaciones sociales condicionadas por las concepciones de género o generacionales aún existentes.

Cuadro 3. Edad mediana a la primera cohabitación y unión, según región de residencia de la mujer

\begin{tabular}{|l|c|c|c|c|}
\hline \multirow{2}{*}{$\begin{array}{l}\text { Región de } \\
\text { residencia }\end{array}$} & \multicolumn{4}{|c|}{ Edad mediana a la primera } \\
\cline { 2 - 5 } & $15-19$ & $20-49$ & $15-49$ & $15-49$ \\
\cline { 2 - 5 } Baja California & 15.75 & 18.37 & 18.21 & 23.19 \\
\hline Coahuila & 15.51 & 18.89 & 18.63 & 22.11 \\
\hline Chihuahua & 15.78 & 18.43 & 18.26 & 22.64 \\
\hline Nuevo León & 15.83 & 19.50 & 19.33 & 22.59 \\
\hline Sonora & 15.93 & 19.36 & 19.15 & 22.86 \\
\hline Tamaulipas & 15.77 & 18.94 & 18.76 & 22.00 \\
\hline Zona Fronteriza & $\mathbf{1 5 . 7 5}$ & $\mathbf{1 8 . 9 4}$ & $\mathbf{1 8 . 7 4}$ & $\mathbf{2 2 . 5 7}$ \\
\hline Resto del país & 15.81 & 19.06 & 18.86 & 23.03 \\
\hline Nacional & $\mathbf{1 5 . 8 0}$ & $\mathbf{1 9 . 0 4}$ & $\mathbf{1 8 . 8 4}$ & $\mathbf{2 2 . 9 5}$ \\
\hline
\end{tabular}

Fuente: Elaboración propia con base en la Encuesta Demográfica Nacional, ENADID 2014

También pueden estar interviniendo en una mayor exposición al riesgo de embarazo, otros aspectos generalmente no destacados en los análisis sociodemográficos, como son los cambios morfológicos y físiológicos a edades cada vez más jóvenes; como el desarrollo corporal y de los genitales, la eyaculación y la aparición de la menarquía condicionan el establecimiento de relaciones sexuales completas y potencialmente propicias para la procreación más temprana a medida que las sociedades alcanzan un mayor desarrollo socioeconómico, lo que puede asociarse a mejores condiciones de nutrición y de salud en general, repercutiendo ello al menos potencialmente, en el aumento de las posibilidades de embarazo en edades más jóvenes (Mercola, 2012).

\section{ARREGLOS CONYUGALES EN EL MARCO DE LOS QUE SE LLEVA A CABO LA SEXUALIDAD Y LA REPRODUCCIÓN}

Los logros alcanzados a través de la lucha social en general, y de las mujeres de manera particular, han incrementado la participación laboral de éstas -así como el divorcio-, atenuando la discriminación por diferente identidad de género y con ello han condicionado el que conjuntamente con los hogares familiares de derecho -que son los dominantes- se presenten otras construcciones familiares entre las que se destacan las familias surgidas por uniones de hecho (también reconocida como unión convivencial de parejas estables); las 
monoparentales conformadas por madres o padres solos; las reconstituidas que surgen de un divorcio, viudez o simple separación.

Entre las familias de hecho se señalan también las homoparentales constituidas por parejas del mismo sexo -lo que es llamado actualmente matrimonios igualitarios- donde la reproducción adopta nuevas formas, ya sea a través de la inseminación artificial o la adopción de hijos, aunque este es aún un proceso incipiente en la región, puesto que al realizarse los cálculos al respecto, se halló que es muy poca la representación de estos hogares. La Encuesta Nacional de Ocupación del año 2016 mostró que sólo el 0.97 por ciento de los habitantes del país se encuentran en dicha situación, sin conocerse aun si hay diferencias en la proporción y el sentido con la región fronteriza al respecto.

\section{INDICADORES DE CAMBIOS EN LA IMPORTANCIA DE LA FAMILIA}

Algunos investigadores sugieren una declinación de importancia en cuanto a las funciones que tradicionalmente se le han atribuido, o posiciones más extremas que sostienen el limitado interés del estudio de la familia como tal pues esta institución responde al modelo patriarcal y nuclear, lo que es controversial de acuerdo al cambiante escenario sociodemográfico (Yépez, 2013).

Al respecto ha existido un amplio debate, planteándose que la pérdida de importancia de la familia se puede documentar a través de diversos aspectos en general ya observados anteriormente en el presente artículo, entre los que destaca:

- Incremento de los nacimientos de niños fuera del matrimonio: En el período que va de 2010 a 2015, se presentó un incremento de cinco por ciento en los eventos referidos.

- Incremento en el porcentaje de madres adolescentes y/o que no están unidas de manera estable: En la zona fronteriza se registran más casos de madres adolescentes, que en el resto del país (Ver Gráfico 3)

Gráfico 3. Porcentaje de madres adolescentes:

Nacional y Promedio Zona Fronteriza (2010-2014)

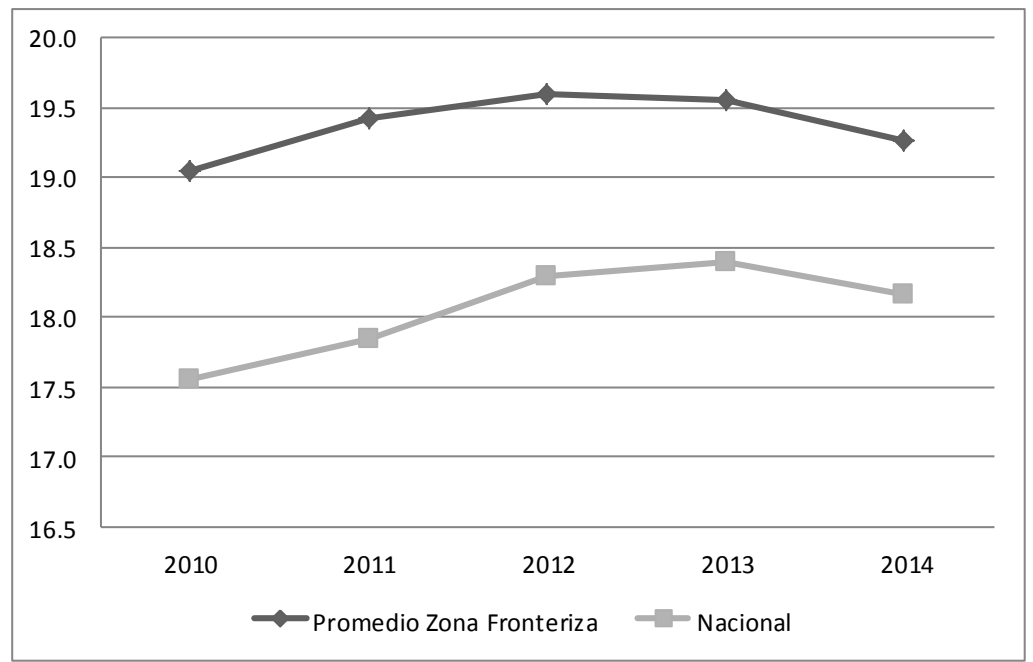

Fuente: Elaboración propia con información de: INEGI, Registros administrativos, Natalidad 
Por otra parte, de los nacimientos registrados en 2014 en la zona fronteriza, 14.3 por ciento fueron de madres que no se encontraban unidas de manera estable, mientras que 10.3 por ciento no especificó su situación conyugal (INEGI, 2017).

- Incremento en el porcentaje de personas divorciadas: el número de divorcios en México se ha incrementado considerablemente en los últimos años, de 2004 a 2015 ha presentado un crecimiento de 83.3 por ciento. Ello está relacionado al incremento de la proporción de hijos viviendo con uno solo de sus padres

Otro aspecto referido en la pérdida de importancia de la familia sería el decremento de tiempo de vida adulta compartida con el cónyuge y sus hijos, lo cual está sin dudas vinculado al mayor tiempo dedicado por las personas a la vida laboral. Ello ha sido tradicionalmente común entre los hombres en edad adulta (30 a 59 años) donde la población económicamente activa generalmente ha superado el 90 por ciento, sin embargo, las mujeres clasificadas como jefas de núcleos presenta actualmente niveles apreciables.

De los trabajos realizados por los jefes de núcleos, la mayor parte $(71.6 \%)$ son empleados $\mathrm{u}$ obreros y una reducida parte son trabajadores por cuenta propia, los que posiblemente podrían contar con más flexibilidad para dedicar tiempo a las labores hogareñas y la atención de los hijos.

También se ha referido en el caso de países desarrollados, matizando en algo la posición anteriormente expuesta, sosteniendo que aunque la estructura de la familia contemporánea está cambiando e inclusive existen indicios en las sociedades avanzadas del debilitamiento de la misma como institución (Pepenoe, 1998) -en la forma tradicionalmente considerada- , no se debe pensar que se está desintegrando.

Así se asume que la familia está perdiendo influencia sobre el comportamiento y opinión de los miembros que los componen y en términos generales, ha venido a ser menos importante en la forma de vida contemporánea, lo cual se refleja en cambios relevantes en la desinstitucionalización interna de los grupos familiares, a través de una mayor autonomía de sus miembros.

En igual sentido se plantea que en la familia se han debilitado en alto grado las funciones que tradicionalmente se desarrollaban, así por ejemplo referente a la función procreadora, se han incrementado las relaciones sexuales previas al matrimonio y con ello las gestaciones extramaritales. También se ha evidenciado el debilitamiento de la función socializadora de los hijos y en el cuidado y manutención de estos ante otras instituciones como las guarderías y las escuelas por la mayor participación de ambos padres en el mercado de trabajo. Ello es de mayor relevancia en los casos de familias monoparentales y/o aquellas encabezadas por mujeres, aspectos que son ampliamente abordados por investigadores de diversos países como son los españoles, a cuyos trabajos es recomendable acudir para profundizar en esta trascendente temática.

De acuerdo a lo planteado, la familia se está debilitando tanto en el sentido de que los grupos familiares individuales están decreciendo en tamaño y se están tornando más inestables, porque la actitud pro familia, como valor cultural, está siendo suplantado por otros ideales como son el desarrollo personal o individual.

Los cambios de actitud y culturales -lo que ha dado en llamarse "la revolución silenciosa" son aspectos condicionantes de la Segunda Transición Demográfica en los que los investigadores hacen gran hincapié. De manera gradual las personas fueron cambiando su visión sobre aspectos sexuales y reproductivos, lo que los hizo más tolerantes a nuevos tipos de conducta como la cohabitación sin intención de casarse o tener hijos, llevar la vida sexual como parejas pero manteniendo residencias diferentes, tener hijo fuera del matrimonio, empleo para las mujeres con hijos en edad escolar y otros aspectos que disminuyeron la 
dependencia de las mujeres y debilitaron las desigualdades de género lo que es destacado desde hace algunas décadas (Salsona, 1997)

Se debe considerar también que las prácticas sexuales en edades tempranas, sin una adecuada protección, pueden tener consecuencias negativas para la sociedad, las familias y los jóvenes, cuando condicionan graves enfermedades de transmisión sexual o la aparición de un embarazo, generalmente no planificado, en contextos donde se percibe esta etapa del ciclo de la vida de las personas como formativa, a través básicamente de la educación institucionalizada. De igual forma resulta de interés como la salud de la joven puede ser afectada a través de una sexualidad y reproducción irresponsable.

Por lo apreciado en la información analizada, la edad al comienzo de las relaciones sexuales entre las mujeres más jóvenes se encuentra entre los 15 y 16 años -en las sexualmente activas- lo que pudiera sugerir que este indicador está descendiendo entre las adolescentes, lo que no parece incidir en un cambio a un nivel más bajo de la edad a la primera unión conyugal o matrimonio formal, pero posiblemente sí en que se eleve la tasa especifica de fecundidad por edad de las adolescentes por una mayor exposición a un embarazo. También llama la atención al respecto la mayor proporción de mujeres que son sexualmente activas en la generalidad de las edades reproductivas en la frontera norte del país (Ver Gráfico 4), lo que expone en un mayor grado a la aparición de embarazos no planificados, particularmente en las primeras relaciones sexuales donde la protección contraceptiva es más limitada (González, H., 2013).

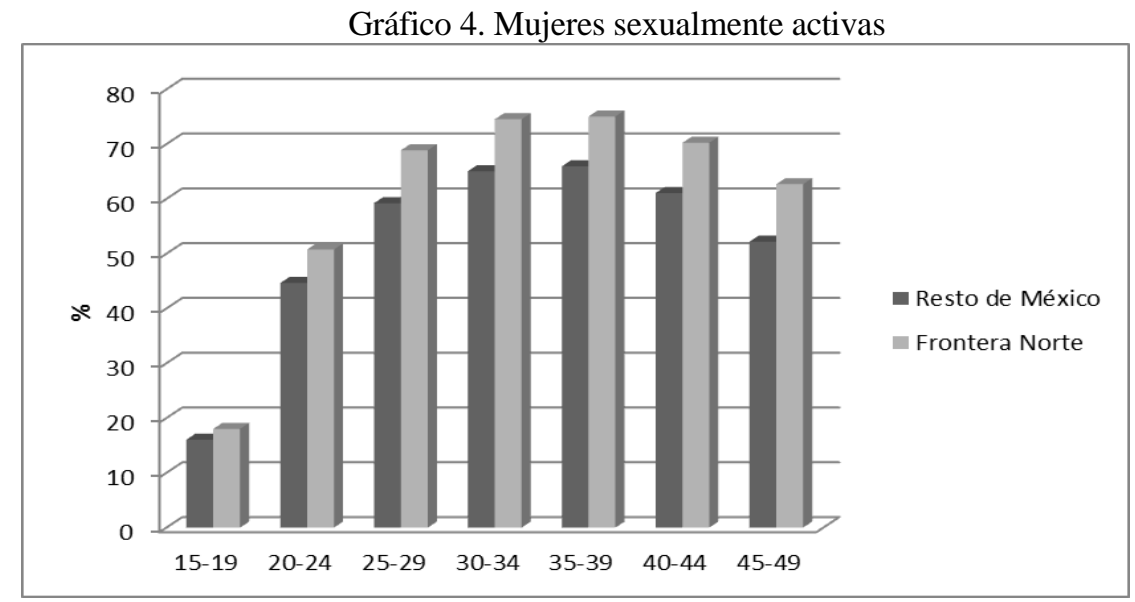

Fuente: INEGI, 2014, Encuesta Nacional de la Dinámica Demográfica

La situaciación conyugal entre las madres adolescentes no presenta grandes variaciones respecto al lugar de residencia, sin embargo, la zona fronteriza exhibe un menor porcentaje de madres adolescentes casadas (17\%) respecto al resto de jóvenes menores de 20 años mexicanas $(19.2 \%)$, lo que puede justificar la existencia de un mayor número de madres adolescentes solteras. 


\section{COMPORTAMIENTO SEXUAL Y REPRODUCCIÓN}

Otro aspecto de interés en el estudio de los jóvenes, vinculado a la sexualidad es la reproducción. La proporción de mujeres adolescentes que han estado embarazadas alcanza casi el 50 por ciento de las mismas, valor que se eleva aún más la zona fronteriza, ello se pudiera explicar en mejor sentido por la mayor sexualidad de las más jóvenes en la frontera. En el grupo de edad 25-29 la fecundidad es generalmente mayor que entre las adolescentes (15-19 años), las que tienen un más elevado peso que las del resto de México.

El no encontrarse estudiando es otro factor que se asocia a la aparición de embarazos entre las jóvenes fronterizas. Según la información aquí analizada (Encuesta Nacional de la Dinámica Demográfica, 2014) del total de jóvenes con nivel inferior al universitario, de las de menos de 25 años de edad el 16.9 han estado embarazadas, mientras que el 41.4 por ciento de las que no cuentan con ningún nivel de escolaridad se han encontrado en dicha situación. Entre las adolescentes los contrastes al respecto son mucho mayores, pues sólo 3.9 por ciento de las que asisten a la escuela han estado embarazadas, proporción que se multiplica más de 14 veces cuando no asisten a las escuelas.

Generalmente se asume que la aparición de un embarazo en una joven tiene en el contexto del país importantes implicaciones sociales, familiares y personales, lo que debe ser matizado en correspondencia con lo expresado por las jóvenes fronterizas al respecto. En este sentido se aprecia que sólo poco más de la décima parte $(10.4 \%)$ de las adolescentes se encontraba estudiando cuando experimentó un primer embarazo, proporción que es menor entre las adolescentes del resto de México (9.0 \% respectivamente), siendo las adolescentes fronterizas las que precisamente dejaron de estudiar en mayor grado cuando apareció la gestación.

Con relación a los embarazos declarados por las jóvenes de menor edad, es posible asumir que la mayor parte sean no planificados y por dicha razón puedan conducir a interrupciones provocadas de las gestaciones o al nacimiento de hijos no deseados. De las mujeres de 15 a 19 años alguna vez embarazadas, poco más de once por ciento ha concluido al menos un embarazo con una interrupción, y aunque no es posible determinar qué proporción de éstas interrupciones han sido intencionales, si es probable considerar que muchos de los abortos voluntarios que experimentan las jóvenes, se realizan en condiciones médicas y sanitarias inadecuadas, debido a la ilegalidad que pesa sobre esta práctica en la zona fronteriza, y/o limitaciones económicas para realizárselo en Estados Unidos por carecer este grupo de recursos para ello, lo que atenta contra la salud y los derechos sexuales y reproductivos de las mujeres jóvenes.

Con independencia de las interrupciones provocadas, así como de los abortos espontáneos y los nacidos muertos, la forma más común en que terminan los embarazos entre las jóvenes fronterizas es un nacido vivo (86.1\%), aspecto que se aborda aquí por ser generalmente considerada la fecundidad como la variable de cambio demográfico más importante.

Entre las interrogantes a las que se ha tratado de dar respuesta en las investigaciones que sobre este tópico se han realizado, desde la sociodemografía, se encuentra el comportamiento peculiar de la fecundidad en las mujeres jóvenes en un contexto de descenso generalizado de los niveles de la referida variable que acompañan a una etapa avanzada de la transición demográfica.

Como se ha observado en países desarrollados y en alguna medida en otros en vías de desarrollo, pero con significativos avances en el proceso demográfico, los niveles de fecundidad de las mujeres más jóvenes pueden experimentar un importante incremento cuando ya ha ocurrido una sustancial caída de los indicadores de fecundidad, incluso hasta 
niveles por debajo del reemplazo poblacional para el resto de las mujeres más adultas (United Nations, 1988,1989).

La referida situación ha sido evidenciada y analizada por algunos investigadores (Chackiel y Schkolnik, 1990; González, 2006) a nivel de países o grandes regiones político administrativas. Menos explorado resulta el estudio de este tópico en contextos de escala menor, como puede ser una localidad urbana, donde los niveles condicionantes e implicaciones de la fecundidad juvenil son aspectos prácticamente desconocidos, a pesar de la trascendencia para las diferentes esferas de la sociedad de contar con información al respecto.

Se puede asumir, que una buena parte de los cambios con relación a la fecundidad en el estado de Baja California, vinculados básicamente a las jóvenes, particularmente a las de 15 a 19 años, quienes han presentado muy elevadas tasas específicas de fecundidad superiores a 150 por mil, superando el valor de la generalidad de las mujeres de los grupos de edad, lo cual es poco común en el resto del país (González,2006), comenzando a disminuir en años más cercanos, dejando de tener el lugar primario que presentaba anteriormente.

Existen planteamientos generalizados, sustentados en elementos empíricos, referidos a que por las características biológicas, psíquicas o socioeconómicas de los jóvenes, estos son más propensos a adquirir enfermedades de transmisión sexual, sufrir los efectos adversos de abortos realizados en condiciones inadecuadas y abusivas, experimentar embarazos no planificados o tener hijos no deseados, por lo que necesitan mayor y más especializada atención en torno a la planificación sexual y reproductiva de los mismos.

Lo observado permite platear la necesidad de llevar a cabo estudios más amplios y concluyentes sobre la familia en esta parte del país para la resolución de problemáticas complejas puesto que la familia representa la unidad básica del comportamiento poblacional ya que los principales factores demográficos son condicionados según los acontecimientos que se producen en el interior de esta (Yépez-Martínez, 2013)

\section{CONCLUSIONES}

A pesar de los retos que implica el desarrollo de estudios sobre la familia en contextos peculiares, como la región de la frontera norte de México, se justifica ampliamente la realización de los mismos por el acercamiento a la comprensión de las problemáticas que ello posibilita y la transmisión posterior de dichas experiencias al resto del país y otras regiones del mundo en desarrollo.

Entre los aspectos que pueden resultar de interés para las temáticas planteadas en este documento, se encuentran los relacionados a características sociodemográficas de las familias jóvenes de la frontera de México y vinculado a ello algunas problemáticas que afectan directamente a este importante sector de la población, particularmente a las mujeres.

Algunas de las características sociodemográficas de la región que aquí se destacan son:

Una mayor proporción de hogares donde residen jóvenes que en el resto del país.

Un menor número de hogares nucleares donde residen jóvenes, y por el contrario más hogares compuestos, unipersonales y con corresidentes.

Entre las problemáticas que afectan a los jóvenes de la Región se señalan:

Una mayor actividad sexual fuera de uniones estables y sin una preparación previa para ello, a través de la educación sexual, lo que ha condicionado una alta incidencia de enfermedades de trasmisión sexual y de embarazos adolescentes no planificados, particularmente entre las mujeres migrantes y las que no estudian. 
Otra condicionante de dicha problemática, determinada en última instancia, por las inequidades que plantean las relaciones de género dominantes en la sociedad en conjunto y no sólo el medio hogareño.

Según lo apreciado en el actual artículo; contrariamente a la visualización tradicional de familia en la actualidad debe prevalecer el amor, la solidaridad y el sentido de convivencia que se contrapongan a los estereotipos sexistas a presupuestos ideológicos y a requerimientos normativos machistas.

Por último, se debe destacar que este es sólo un esfuerzo muy primario en el estudio de las familias jóvenes y sus problemáticas, busca llamar la atención al respecto y motivar la realización de análisis más sistemáticos y profundos sobre esta importante institución al interior de la que se gestan los cambios socioculturales y demográficos de las diferentes regiones en el mundo, de las que se particularizan algunas como la frontera norte de México.

\section{BIBLIOGRAFÍA}

Colegio de La Frontera Norte (2006) Encuesta de Salud Reproductiva de los Adolescentes en Baja California. Tijuana, México.

Chaquiel, J. y Schkolnik, S. (1992) "La transición de la fecundidad en América Latina". Notas de Población, 20(55): 161-192.

González, H. (2006) "Cambios previsibles en los niveles de fecundidad de las adolescentes mexicanas. El caso de la frontera norte". Revista Frontera Norte, 18 (36): 29-52.

González, H. (2013) "La reproducción de las jóvenes de la frontera norte de México. Niveles territoriales y factores condicionantes". Revista Estudios Fronterizos, 14 (27): 65-96.

Instituto Nacional de Geografía e Informática (INEGI). (2002) Censo General de Población y Viviendas, 2000. Aguas Calientes, México.

INEGI (2009) Encuesta Nacional de la Dinámica Demográfica. México.

INEGI (2014) Encuesta Nacional de la Dinámica Demográfica (ENADID). México

INEGI (2015) Encuesta Intercensal (EIC). México.

Mercola, J. (2012) Causas de la pubertad temprana: ¿Por qué ahora es normal? En Mercola.com, accesible en https://goo.gl/OR4l6

Pepenoe, D. (1998) Disturbing the nest. Family change and decline in modern societies. Nueva York: Aldine de Gruyter.

Salsona, M. (1997) Las transformaciones familiares recientes desde la perspectiva de género. Barcelona: Universidad Autónoma de Barcelona.

United Nations, Department of International Economic and Social Affairs. Population Division. (1988) “Adolescent Reproductive Behaviour. Evidence from Developed Countries". Population Studies, I: 178.

United Nations, Department of International Economic and Social Affairs. Population Division. (1989) "Adolescent Reproductive Behaviour. Evidence from Developing Countries", Population Studies, II: 128.

Vargas, E. y Navarro, A. (2013) "La estructura de la jefatura de los hogares de la frontera norte en la última década". Estudios Fronterizos, 14(27): 123-150.

Yépez, B. (2013) "La demografía de la familia y los hogares". Universidad Central de Venezuela, Cuadernos de Cendes, 30 (83): 121-133. 


\section{Breve currículo:}

\section{Humberto González Galbán}

Realizó sus estudios universitarios en La Habana y de Maestría en Demografía y Doctorado en Ciencias Sociales en Baja California. Comenzó trabajando en la Oficina Nacional del Censo de Cuba. Se incorporó en el año 2001 al Colegio de la Frontera Norte, en México, donde ocupa actualmente la plaza de Investigador Titular C, siendo miembro del Sistema Nacional de Investigación. Ha impartido cursos sobre Demografía en varios países, publicado decenas de artículos en países de América Latina y Europa. En el 2014 alcanzó, junto a otros colegas del departamento de Estudios de Población el Premio Nacional de Demografía del 2014.

\section{Adriana Ortiz Peredo}

Licenciada en Economía por la Universidad Veracruzana. Maestra en Demografía por el Colegio de la Frontera Norte. Actualmente es Técnica Académica en el Departamento de Estudios de Población en El Colegio de la Frontera Norte. Sus Áreas de interés son: envejecimiento poblacional, morbilidad de la población adulta mayor, fecundidad y salud reproductiva. 